\title{
Chloroplast DNA Diversity in Prunus and Its Implication on Genetic Relationships
}

\author{
Mariem Bouhadida ${ }^{1}$ \\ Departamento de Pomología, Estación Experimental de Aula Dei, CSIC, Apdo 202, 50080 Zaragoza, Spain
}

Juan P. Martín

Departamento de Biología Vegetal, Escuela Técnica Superior de Ingenieros Agrónomos, Universidad Politécnica de Madrid, Ciudad Universitaria s/n, 28040 Madrid, Spain

Gemady Eremin

Krymsk Breeding Station, Krymsk, Krasnodar Region, 353330 Russian Federation

Jorge Pinochet

Agromillora Catalana, S.A., El Rebato s/n, 08739 T.M Subirats, Barcelona, Spain

\author{
María Á. Moreno and Yolanda Gogorcena \\ Departamento de Pomologiá, Estación Experimental de Aula Dei, CSIC, Apdo 202, 50080 Zaragoza, Spain
}

\begin{abstract}
Additional INDEX words. cpDNA haplotypes, PCR-RFLP, restriction enzymes, universal primers
Abstract. Chloroplast DNA (cpDNA) in 84 Prunus L. accessions (interspecific hybrids and Prunus species) were analyzed to confirm the maternal origin of the interspecific hybrids of Prunus and to establish genetic relationships among Prunus species. The polymerase chain reaction-restriction fragment length polymorphism (PCR-RFLP) method, which included amplification of cpDNA regions with three universal primer pairs (K1K2, HK, DT) and subsequent digestion with three restriction enzymes (AluI, HinfI, TaqI), revealed 33 haplotypes for the 84 accessions studied. Fourteen from these cpDNA haplotypes were shared by two or more accessions, and 19 were unique. Accessions sharing the same haplotype have maternal relationships among them, which allowed identity confirmation of maternal progenitors of Prunus interspecific hybrids in these cases. Unweighted pair group method average (UPGMA) and minimum-length spanning tree (MST) analyses were performed based on shared common fragments and the number of mutational differences among the 33 haplotypes, respectively. The cpDNA polymorphisms detected made possible the analysis of genetic relationships among the studied Prunus accessions. Most of the recovered relationships are in agreement with current taxonomic hypotheses and artificial crosses.
\end{abstract}

Prunus (subfamily Prunoideae of the family Rosaceae) is a large and diverse genus comprising $\approx 400$ species of trees and shrubs. This genus originated chiefly in the Northern hemisphere and is widely represented in Europe (Dosba et al., 1994). The basic chromosome number of Prunus is $x=8$. Almond [ $P$. dulcis D.A. Webb (formerly $P$. amygdalus Batsch)], peach $[P$. persica (L.) Batsch], apricot (P. armeniaca L.), and sweet cherry $(P$. avium $\mathrm{L}$.) are diploids with $2 n=2 x=16$. Tetraploid species include sloe $(P$. spinosa $\mathrm{L}$.), sour cherry $(P$. cerasus L.), and $P$. fruticosa Pall. $(2 n=4 x=32)$ and hexaploid species are represented by European plums $[P$. domestica $\mathrm{L}$. and $P$. insititia L. $(2 n=6 x=48)]$. Prunus is economically important because of

Received for publication 12 Jan. 2007. Accepted for publication 27 June 2007. This research was funded by CICYT (Comisión Interministerial de Ciencia y Tecnología, AGL2002-04219 and AGL 2005-05533), Bilateral Spain-France (HF03-273) and DGA (A28, A44) projects, and cofunded by the European Regional Development Fund. M.B. was supported by a fellowship from the AECI (Agencia Española de Cooperación Internacional) of the Spanish Ministry of Foreign Affairs.

The authors thank Dr. P. Catalán for helpful comments on the manuscript and contribution in the analyses. We appreciate the collaboration of A. Zanetto (INRA, Bordeaux) and H. Duval (INRA, Avignon) for providing us some plant material used in this study. We gratefully acknowledge M.L. Cunill (Agromillora Catalana S.A.) for plant material management.

${ }^{1}$ Corresponding authors: Mariem Bouhadida and Yolanda Gogorcena. E-mail: mbouhadida@hotmail.com and aoiz@eead.csic.es. its diversified uses as fruit [e.g., European plums, japanese plum (P. salicina Lindl.), peach, apricot, sour cherry, sweet cherry, and almond], ornamentals, forestry, or for industrial purposes (Webster, 2005). The presence of a large number of Prunus species increases the possibilities of interspecific hybridization and makes the establishment of botanical classification quite complicated (Layne, 1987). Traditional taxonomy of Prunus has used morphology to estimate relationships among the species in question and to provide taxonomy for such genus. Systematic classification in Prunus is still controversial. De Tournefort (1700) recognized six distinct genera within Prunus "sensu lato" based on fruit morphology: Amygdalus L., Armeniaca Mill., Cerasus Mill., Laurocerasus Duhamel, Persica Mill., and Prunus "sensu stricto". Bentham and Hooker (1865) were the first to unite the six genera of De Tournefort (1700) into a single genus Prunus, which was subdivided into seven sections: Amygdalopsis, Amygdalus, Armeniaca, Cerasoides, Cerasus, Laurocerasus, and Prunus. Koehne (1893) initially divided the genus Prunus into seven subgenera, but later (Koehne, 1911) recognized only four: Amygdalus, Cerasus, Padus, and Prunophora (= Prunus). Rehder (1940) reviewed the previous treatments and divided the genus into five subgenera: Prunus [= Prunophora $($ Neck.)] Focke, Amygdalus (L.) Benth. Hook., Cerasus (Adans.) Focke, Padus (Moench) Focke, and Laurocerasus (Ser.) Rehd. The subgenus Cerasus, as defined by 
Rehder, was composed of a large and diverse group of species and was later divided by Ingram (1948) into subgenera Cerasus and Lithocerasus Ingram. At present, biochemical (isoenzyme) and molecular (DNA analysis) approaches allow a more accurate estimate of genetic affinities and evolutionary relatedness among Prunus taxa that permits a comparison with traditional classifications established by taxonomists. Several investigations were carried out to assess genetic variation among Prunus species using isozyme markers (Arulsekar et al., 1986; Mowrey and Werner, 1990). Molecular markers such RFLPs, RAPDs, and SSRs were also used for phylogenetic and genetic diversity studies among Prunus species (Casas et al., 1999; Kaneko et al., 1986; Rohrer et al., 2004).

Noncoding regions of cpDNA have been explored under the assumption that these regions should be under less functional constraint than coding regions and should hence provide greater levels of variation for phylogenetic analyses (Gielly and Taberlet, 1994). Universal primers (Demesure et al., 1995; Dumolin-Lapegue et al., 1997; Taberlet et al., 1991) have been used to amplify fragments of noncoding regions of cpDNA followed by digestion with restriction enzymes. Fragment length polymorphism was consequently revealed within the amplified fragments (Demesure et al., 1996; El Mousadek and Petit, 1996; King and Ferris, 1998). This PCR-RFLP method, also known as cleaved amplified polymorphic sequences (CAPS) (Konieczny and Ausubel, 1993), is a readily accessible laboratory technique that can be used to evaluate large portions of the chloroplast genome in numerous individuals in a short time and at a lower cost. This method has been used to analyze phylogenetic relationships of cultivated Prunus species (Badenes and Parfitt, 1995; Panda et al., 2003) and to determine the phylogeography and the population diversity in $P$. spinosa and P. avium (Mohanty et al., 2000, 2001, 2002).

Current trends in breeding stone fruit rootstocks are based on the production of interspecific hybrids, aiming at putting together favorable traits that appear in different species (Moreno, 2004). Commercial Prunus rootstocks that are a result of uncontrolled interspecific pollinations are available on the market. Moreover, the pedigree of most of the clones is unknown due to the lack of parental control, and this can be a major constraint for their use in breeding programs. It is subsequently desirable to appropriately characterize the parentage of the rootstock material as a means to evaluate the success of various crosses.

In this study, a characterization of Prunus species and rootstocks (interspecific hybrids) on the basis of cpDNA screening was carried out with the following objectives: 1) to analyze the genetic diversity of interspecific hybrids and species belonging to the Prunus genus; 2) to test the usefulness of the method to confirm the maternal origin of Prunus interspecific hybrids; 3) to investigate genetic relationships among Prunus species. The characterization of this plant material and the verification of its pedigree before it is introduced into the breeding programs of the Experimental Station of Aula Dei at Zaragoza, Spain, will be useful to increase the genetic background in these programs.

\section{Materials and Methods}

Plant materials. Sixty-two accessions of various interspecific hybrids of the genus Prunus were obtained from the Krymsk Experimental Breeding Station (KEBS) in Russia including pedigree data (see Table 1). These accessions form a group highly representative of the genetic variability in the KEBS collection. Twenty-two Prunus species were sampled from three important collections of Prunus in Europe: KEBS; Estación Experimental de Aula Dei-CSIC at Zaragoza, Spain; Institut Nationale de Recherche Agronomique (INRA) at Bordeaux and Avignon, France (Table 1). Fresh leaves were collected from plants, frozen, and stored at $-80{ }^{\circ} \mathrm{C}$.

DNA EXTRACTION, AMPLIFICATION, AND DIGESTION. DNA was extracted from frozen leaves following the cetyltrimethylammonium bromide (CTAB) extraction method described by Cheng et al. (1997). DNA quality was examined by electrophoresis in $0.8 \%(\mathrm{w} / \mathrm{v})$ agarose gels, and DNA concentration was quantified using a spectrophotometer. Extracted DNA was diluted to $5 \mathrm{ng} \cdot \mu \mathrm{L}^{-1}$ (working solution) and stored at $-20{ }^{\circ} \mathrm{C}$ until PCR reactions.

Initially, amplifications of cpDNA were done using five universal primer pairs (Table 2) described by DumolinLapegue et al. (1997). PCR reactions were performed in a final volume of $40 \mu \mathrm{L}$ containing $20 \mathrm{ng}$ of template DNA, $0.2 \mathrm{~mm}$ of each of the four dNTPs, $0.2 \mu \mathrm{M}$ of forward and reverse primers each, $2 \mathrm{mM} \mathrm{MgCl}_{2}$, and $1 \mathrm{U}$ of $P f u$ DNA polymerase in the buffer provided by the manufacturers of the enzyme (Biotools, B\&M Laboratories, S.A., Madrid, Spain). Amplifications were carried out in a Gene Amp 2700 thermocycler (Applied Biosystems, Foster City, CA) using the following temperature cycles: 1 cycle of $3 \mathrm{~min}$ at $95^{\circ} \mathrm{C}, 40$ cycles of $1 \mathrm{~min}$ at $94{ }^{\circ} \mathrm{C}$, $45 \mathrm{~s}$ at $52-62{ }^{\circ} \mathrm{C}$, and $2-5 \mathrm{~min} 30 \mathrm{~s}$ at $72{ }^{\circ} \mathrm{C}$ (annealing temperatures and extension times for each primer pair are provided in Table 2). The last cycle was followed by a final incubation for $10 \mathrm{~min}$ at $72{ }^{\circ} \mathrm{C}$, and then the PCR products were stored at $4{ }^{\circ} \mathrm{C}$ before analysis. Amplification of cpDNA products was checked by electrophoresis on $1 \%(\mathrm{w} / \mathrm{v})$ agarose gels, stained with ethidium bromide, and visualized under ultraviolet (UV) light. Approximate amplified fragment sizes were estimated with a 1-kb ladder DNA marker (Invitrogen, Carlsbad, CA).

Amplified fragments were digested with three restriction enzymes: AluI, Hinfl, and TaqI (Invitrogen). The reaction mixture was incubated overnight at $37^{\circ} \mathrm{C}$ with $3 \mathrm{U}$ of $A l u \mathrm{I}$ or HinfI, or $1 \mathrm{~h}$ at $65^{\circ} \mathrm{C}$ with $5 \mathrm{U}$ of TaqI. Restriction products were separated on $2.5 \%(\mathrm{w} / \mathrm{v})$ agarose gels, run in a Tris-borateEDTA buffer $(1 \times)$ for $5 \mathrm{~h}$ at $3 \mathrm{~V} \cdot \mathrm{cm}^{-1}$, then stained with ethidium bromide and visualized under UV light. Restriction fragment sizes were estimated with a 50-bp ladder marker (Amersham, Piscataway, NJ).

DAtA ANALysis. The presence or absence of each restriction fragment at each polymorphic site was scored as binary data and used to identify chloroplast haplotypes. Similarity between pairs of haplotypes was estimated from the binary matrix using the simple matching coefficient. The similarity matrix was employed to construct a dendrogram by unweighted pair group method average (UPGMA). All the analyses were computed using the programs from the NTSYS-pc package, version 2.1 (Rohlf, 2000).

Additionally, a minimum-length spanning tree between haplotypes of interspecific hybrids and Prunus parents was performed using the programs from the NTSYS-pc, version 2.1 (Rohlf, 2000). The procedure consists of calculating the number of mutational differences between haplotypes and then connecting them by direct links of the smallest possible length (Prim, 1957). 
Table 1. List of 84 accessions (interspecific hybrids and species) of Prunus studied. ${ }^{z}$

\begin{tabular}{|c|c|c|c|c|c|}
\hline Accession & Origin $^{y}$ & Genotype (female $\times$ male $)$ & Section $^{\mathrm{x}}$ & Subgenus $^{\mathrm{x}}$ & Haplotype \\
\hline PACE00-03 & KEBS & $(P$. pumila $\times P$. armeniaca $) \times P$. domestica & Microcerasus & Cerasus & H1 \\
\hline PACE00-08 & KEBS & $(P$. pumila $\times P$. armeniaca $) \times P$. domestica $(4 x)$ & Microcerasus & Cerasus & H1 \\
\hline PACE01-26 & KEBS & $(P$. besseyi $\times P$. salicina $) \times(P$. cerasifera $\times P$. persica $)$ & Microcerasus & Cerasus & H1 \\
\hline PACE01-46 & KEBS & $(P$. pumila $\times P$. armeniaca $\times P$. domestica $)$ & Microcerasus & Cerasus & $\mathrm{H} 1$ \\
\hline PACE02-14 & KEBS & $(P$. pumila $\times P$. salicina $) \times(P$. cerasifera $\times P$. persica $)$ & Microcerasus & Cerasus & $\mathrm{H} 1$ \\
\hline PADAC01-47 & KEBS & (P. pumila $\times P$. armeniaca $\times P$. domestica $)$ & Microcerasus & Cerasus & H1 \\
\hline EVRICA & KEBS & $(P$. besseyi $\times P$. salicina $) \times P$. cerasifera $($ Otletchniza $)$ & Microcerasus & Cerasus & H1 \\
\hline Krasnaia-lenta & KEBS & $(P$. besseyi $\times P$. salicina $) \times P$. cerasifer $a=$ Hiawatha $\times P$. cerasifera & Microcerasus & Cerasus & H1 \\
\hline Krasni bordur & KEBS & $(P$. besseyi $\times P$. salicina $) \times P$. cerasifera $($ Pissardii $)$ & Microcerasus & Cerasus & H1 \\
\hline ST-N1 & KEBS & $P$. besseyi $\times P$. cerasifera & Microcerasus & Cerasus & H1 \\
\hline ST-N2 & KEBS & $P$. besseyi $\times P$. cerasifera & Microcerasus & Cerasus & H1 \\
\hline $\mathrm{AD}-3$ & EEAD & P. bessseyi & Microcerasus & Cerasus & H1 \\
\hline PACE02-08 & KEBS & $(P$. salicina $\times P$. cerasifera $) \times(P$. armeniaca $\times P$. persica $)$ & Euprunus & Prunophora & $\mathrm{H} 2$ \\
\hline PACE02-21 & KEBS & $\begin{array}{l}{[P \text {. salicina }(\text { Santa Rosa }) \times P \text {. cerasifera }(4 x)] \times[P \text {. cerasifera }} \\
\quad \times P \text {. persica }(\text { Kuban }-86)(4 x)]\end{array}$ & Euprunus & Prunophora & $\mathrm{H} 2$ \\
\hline $\mathrm{AD}-11$ & EEAD & P. salicina & Euprunus & Prunophora & $\mathrm{H} 2$ \\
\hline PACE00-09 & KEBS & $P$. cerasifera $\times P$. persica $(4 x)$ & Euprunus & Prunophora & H3 \\
\hline PACE01-38 & KEBS & P. dasycarpa $\times P$. domestica & Armeniaca & Prunophora & $\mathrm{H} 3$ \\
\hline PACE01-39 & KEBS & P. dasycarpa $\times P$. spinosa, $\mathrm{F} 2$ & Armeniaca & Prunophora & $\mathrm{H} 3$ \\
\hline PACE01-40 & KEBS & P. dasycarpa $\times P$. spinosa, $\mathrm{F} 2$ & Armeniaca & Prunophora & $\mathrm{H} 3$ \\
\hline PACE01-41 & KEBS & P. dasycarpa $\times$ P. spinosa, $\mathrm{F} 2$ & Armeniaca & Prunophora & H3 \\
\hline PACE02-17 & KEBS & $P$. cerasifera $\times P$. persica (nectarine) & Euprunus & Prunophora & $\mathrm{H} 3$ \\
\hline PACE02-18 & KEBS & P. cerasifera $\times P$. persica $[$ Krymsk-86 $(4 x)]$ & Euprunus & Prunophora & H3 \\
\hline $\mathrm{AD}-16$ & INRA-B & P. fruticosa & Eucerasus & Cerasus & $\mathrm{H} 4$ \\
\hline AP-2 & KEBS & $P$. cerasifera $\times P$. persica (nectarine) & Euprunus & Prunophora & H5 \\
\hline Krymsk-1 & KEBS & $P$. cerasifera $\times P$. tomentosa & Euprunus & Prunophora & H5 \\
\hline PACE02-15 & KEBS & P. cerasifera $\times(P$. communis $\times P$. bucharica $)$ & Euprunus & Prunophora & H5 \\
\hline PACE01-42 & KEBS & P. kansuensis $\times$ P. davidiana $\times$ P. persica & Euamygdalus & Amygdalus & H6 \\
\hline PACE01-43 & KEBS & P. kansuensis $\times P$. davidiana $\times P$. persica & Euamygdalus & Amygdalus & H6 \\
\hline AD-19 & INRA-A & P. kansuensis & Euamygdalus & Amygdalus & H6 \\
\hline PACE02-10 & KEBS & $P$. brigantiaca $\times P$. persica & Armeniaca & Prunophora & $\mathrm{H} 7$ \\
\hline PACE02-11 & KEBS & $P$. brigantiaca $\times P$. persica & Armeniaca & Prunophora & $\mathrm{H} 7$ \\
\hline AD-20 & INRA-A & P. brigantiaca & Armeniaca & Prunophora & $\mathrm{H} 7$ \\
\hline PACE01-24 & KEBS & $P$. americana $\times P$. persica & Prunocerasus & Prunophora & $\mathrm{H} 8$ \\
\hline PACE02-09 & KEBS & P. americana $\times P$. persica & Prunocerasus & Prunophora & $\mathrm{H} 8$ \\
\hline PACE02-12 & KEBS & $P$. americana $\times P$. persica & Prunocerasus & Prunophora & $\mathrm{H} 8$ \\
\hline PACE00-02 & KEBS & P. tenella $\times P$. dulcis & Chameamygdalus & Amygdalus & H9 \\
\hline PACG00-02 & KEBS & P. nana $\times$ P. dulcis & Chameamygdalus & Amygdalus & H9 \\
\hline AD-13 & EEAD & P. tenella & Chameamygdalus & Amygdalus & H9 \\
\hline PACE01-30 & KEBS & P. cerasifera $\times P$. bucharica & Euprunus & Prunophora & $\mathrm{H} 10$ \\
\hline PACE01-31 & KEBS & P. cerasifera $\times P$. bucharica & Euprunus & Prunophora & $\mathrm{H} 10$ \\
\hline PACE01-33 & KEBS & P. cerasifera $\times P$. kalmycovii & Euprunus & Prunophora & $\mathrm{H} 10$ \\
\hline PACE00-12 & KEBS & P. spinosa $\times(P$. cerasifera $\times P$. persica $)($ Kuban -1$)$ & Euprunus & Prunophora & H11 \\
\hline PACE01-27 & KEBS & $P$. spinosa $\times(P$. cerasifera $\times P$. persica $)$ & Euprunus & Prunophora & H11 \\
\hline PACE00-07 & KEBS & $P$. spinosa $\times P$. persica $(4 \mathrm{x})$ & Euprunus & Prunophora & H11 \\
\hline
\end{tabular}


Table 1. Continued.

\begin{tabular}{|c|c|c|c|c|c|}
\hline Accession & Origin $^{y}$ & Genotype (female $\times$ male $)$ & Section $^{\mathrm{x}}$ & Subgenus $^{\mathrm{x}}$ & Haplotype \\
\hline$\overline{\mathrm{AD}}-2$ & EEAD & P. armeniaca & Armeniaca & Prunophora & H12 \\
\hline AD-9 & EEAD & P. persica & Euamygdalus & Amygdalus & $\mathrm{H} 13$ \\
\hline PACEOR-03 & KEBS & $\begin{array}{l}{[(P . \text { americana } \times P \text {. simonii }) \times P \text {. spinosa }]} \\
\quad \times(P . \text { cerasifera } \times P \text {. persica })\end{array}$ & Prunocerasus & Prunophora & H14 \\
\hline Vesenia plamiya & KEBS & (P. americana $\times P$. simonii $) \times P$. cerasifera $($ Pissardii) & Prunocerasus & Prunophora & H14 \\
\hline $\mathrm{AD}-18$ & INRA-B & P. americana & Prunocerasus & Prunophora & H14 \\
\hline PACE02-04 & KEBS & P. kansuensis $\times P$. persica (Red leaf) & Euamygdalus & Amygdalus & H17 \\
\hline PACE01-37 & KEBS & P. cerasifera $\times P$. prostrata, $\mathrm{F} 2$ & Euprunus & Prunophora & H18 \\
\hline PACE01-45 & KEBS & P. prostrata $\times P$. cerasifera $\times P$. domestica & Microcerasus & Cerasus & H18 \\
\hline PACE00-10 & KEBS & P. cerasifera $\times P$. bucharica & Euprunus & Prunophora & H19 \\
\hline AD-14 & EEAD & $(P$. besseyi $\times P$. salicina $)($ Sapa $)$ & Microcerasus & Cerasus & $\mathrm{H} 20$ \\
\hline PACE00-06 & KEBS & $\begin{array}{l}(P . \text { besseyi } \times P \text {. salicina })(\text { Sapa }) \times(P \text {. cerasifera } \\
\quad \times P \text {. tomentosa })(\text { Krymsk }-1)\end{array}$ & Microcerasus & Cerasus & $\mathrm{H} 20$ \\
\hline AD-17 & EEAD & P. mahaleb & Mahaleb & Cerasus & $\mathrm{H} 22$ \\
\hline $\mathrm{AD}-4$ & EEAD & P. cerasifera & Euprunus & Prunophora & $\mathrm{H} 23$ \\
\hline AD-8 & EEAD & P. insititia & Euprunus & Prunophora & $\mathrm{H} 24$ \\
\hline L-2 & KEBS & P. lannesiana & Pseudocerasus & Cerasus & $\mathrm{H} 25$ \\
\hline $\mathrm{AD}-22$ & EEAD & P. bucharica & Euamygdalus & Amygdalus & $\mathrm{H} 26$ \\
\hline $\mathrm{AD}-12$ & EEAD & P. spinosa & Euprunus & Prunophora & $\mathrm{H} 27$ \\
\hline $\mathrm{AD}-10$ & EEAD & P. pumila & Microcerasus & Cerasus & $\mathrm{H} 28$ \\
\hline AD-7 & EEAD & P. incana & Microcerasus & Cerasus & $\mathrm{H} 29$ \\
\hline AD-15 & INRA-B & P. davidiana & Euamygdalus & Amygdalus & $\mathrm{H} 30$ \\
\hline AD-5 & EEAD & P. dasycarpa & Armeniaca & Prunophora & H31 \\
\hline AD-6 & EEAD & P. domestica & Euprunus & Prunophora & H32 \\
\hline AD-1 & EEAD & P. dulcis & Euamygdalus & Amygdalus & H33 \\
\hline
\end{tabular}

${ }^{\mathrm{z}}$ Classification into sections and subgenera is according to Rehder (1940). Haplotypes found in accessions are also included.

${ }^{y}$ KEBS, Krymsk Experimental Breeding Station, Russia; EEAD, Estación Experimental de Aula Dei-CSIC, Zaragoza, Spain; INRA-A, Institut Nationale de Recherche Agronomique (INRA), Avignon, France; INRA-B, INRA, Bordeaux, France.

${ }^{x}$ Section and subgenus given here correspond to the first species, representing female parent or maternal progenitor for the interspecific hybrid accessions.

\section{Results}

Chloroplast DNA restriction patterns. Polymorphisms in noncoding regions of the chloroplast DNA were studied in 84 interspecific hybrids and species of the genus Prunus. Initially, five universal primer pairs of cpDNA were used for amplification. Thereafter, and due to their high degree of amplification (Table 2), three primer pairs (DT, HK, K1K2) were chosen for this study. Estimated sizes of the fragments amplified with the

Table 2. Chloroplast DNA (cpDNA) universal primer pairs used for the amplification reactions in Prunus accessions; polymerase chain reaction (PCR) conditions, estimated size of amplified fragments, and quality of amplification are shown.

\begin{tabular}{|c|c|c|c|c|}
\hline \multirow[b]{2}{*}{$\begin{array}{l}\text { Abbreviation of } \\
\text { cpDNA primers }\end{array}$} & \multicolumn{2}{|c|}{ PCR conditions } & \multirow[b]{2}{*}{$\begin{array}{c}\text { Amplified } \\
\text { fragment (bp) }\end{array}$} & \multirow[b]{2}{*}{$\begin{array}{c}\text { Degree of } \\
\text { amplification }^{\mathrm{y}}\end{array}$} \\
\hline & $\begin{array}{l}\text { Annealing } \\
\text { temp }\left({ }^{\circ} \mathrm{C}\right)\end{array}$ & Extension time & & \\
\hline DT & 52 & $2 \min 30 s$ & 1150 & ++ \\
\hline $\mathrm{HK}$ & 62 & $3 \mathrm{~min}$ & 1700 & ++ \\
\hline $\mathrm{K} 1 \mathrm{~K} 2$ & 57 & $4 \mathrm{~min}$ & 2650 & ++ \\
\hline $\mathrm{CD}$ & 57 & $5 \min 30 \mathrm{~s}$ & 3800 & + \\
\hline VL & 56 & $5 \mathrm{~min} 30 \mathrm{~s}$ & 3900 & NA \\
\hline
\end{tabular}

${ }^{\mathrm{z}}$ Abbreviations of cpDNA primers as described by Dumolin-Lapegue et al. (1997).

$\mathrm{y}_{++}$, Good amplification; +, weak amplification; NA, no amplification.
DT, HK, and K1K2 primer pairs were 1150,1700 , and $2650 \mathrm{bp}$, respectively. Total size of these fragments represent $\approx 4 \%$ of the total chloroplast genome (considering that cpDNA size in most Prunus spp. is $\approx 140 \mathrm{kbp}$ (Kaneko et al., 1986; Uematsu et al., 1991). No length polymorphisms were detected in the obtained PCR products using any of the primer pairs tested. Amplification products were digested by three restriction enzymes (AluI, Hinfl, and $T a q \mathrm{I})$. From the initial nine primer pair-restriction enzyme combinations, eight (DT-AluI, DTHinfl, DT-TaqI, HK-AluI, HKHinfl, K1K2-AluI, K1K2-Hinfl, and $\mathrm{K} 1 \mathrm{~K} 2-\mathrm{Taq} \mathrm{I})$ gave polymorphic patterns (Table 3). The HK-TaqI combination showed a monomorphic pattern.

These eight primer-enzyme combinations generated 23 polymorphic sites with 42 length mutations (indel mutations) and seven point mutations. Three primer-enzyme combinations (DT-AluI, HK-Hinfl, and K1K2-AluI) showed two polymorphic sites, while DT-Hinfl, DT$T a q \mathrm{I}$, and $\mathrm{HK}-A l u \mathrm{I}$ presented three, 
Table 3. Major patterns and variants (in $b p$ ) of fragments revealed in each polymorphic site detected with different primer pairrestriction enzyme combinations in 84 accessions of hybrids and parents of Prunus studied.

\begin{tabular}{|c|c|}
\hline Polymorphic sites & $\begin{array}{c}\text { Length or point mutations } \\
{\left[\text { major pattern } \rightarrow \text { variant }^{\mathrm{z}}(\mathrm{bp})\right]}\end{array}$ \\
\hline$\overline{\mathrm{DT}}-(A l u \mathrm{I}) 1$ & $675(\mathrm{~B}) \rightarrow 720(\mathrm{~A})$ \\
\hline $\mathrm{DT}-(\operatorname{Alu\mathrm {I}}) 2$ & $\begin{array}{l}475(\mathrm{~B}) \rightarrow 490(\mathrm{~A}), 460(\mathrm{C}), \\
250+225(\mathrm{D})\end{array}$ \\
\hline DT-(Hinfl $) 1$ & $305(\mathrm{~B}) \rightarrow 315(\mathrm{~A}), 175+140(\mathrm{C})$ \\
\hline $\mathrm{DT}-($ Hinfl $) 2$ & $260(\mathrm{C}) \rightarrow 275(\mathrm{~B}), 305(\mathrm{~A})$ \\
\hline DT $-($ Hinfl $) 3$ & $255(\mathrm{~A}) \rightarrow 250(\mathrm{~B})$ \\
\hline $\mathrm{DT}-(\operatorname{Taq} \mathrm{I}) 1$ & $550(\mathrm{~B}) \rightarrow 555(\mathrm{~A}), 540(\mathrm{C})$ \\
\hline $\mathrm{DT}-(\operatorname{Taq} \mathrm{I}) 2$ & $395(\mathrm{C}) \rightarrow 400(\mathrm{~B}), 375(\mathrm{D}), 430(\mathrm{~A})$ \\
\hline $\mathrm{DT}-(\operatorname{Taq} \mathrm{I}) 3$ & $140(\mathrm{~B}) \rightarrow 150(\mathrm{~A})$ \\
\hline $\mathrm{HK}-(A l u \mathrm{I}) 1$ & $560(\mathrm{~B}) \rightarrow 570(\mathrm{~A})$ \\
\hline $\mathrm{HK}-(A l u \mathrm{I}) 2$ & $\begin{array}{r}385(\mathrm{E}) \rightarrow 405(\mathrm{C}), 375(\mathrm{~F}), 350(\mathrm{G}) \\
415(\mathrm{~A}), 410(\mathrm{~B}), 400(\mathrm{D}), 310(\mathrm{H})\end{array}$ \\
\hline $\mathrm{HK}-(A l u \mathrm{I}) 3$ & $65+45(\mathrm{~B}) \rightarrow 110(\mathrm{~A})$ \\
\hline $\mathrm{HK}-(\operatorname{Hinfl}) 1$ & $\begin{array}{l}690(\mathrm{C}) \rightarrow 680(\mathrm{D}), 660(\mathrm{E}), 640(\mathrm{~F}) \\
710(\mathrm{~A}), 700(\mathrm{~B})\end{array}$ \\
\hline $\mathrm{HK}-(\operatorname{Hinfl}) 2$ & $240(\mathrm{C}) \rightarrow 245(\mathrm{~B}), 250(\mathrm{~A})$ \\
\hline $\mathrm{K} 1 \mathrm{~K} 2-(A l u \mathrm{I}) 1$ & $425(\mathrm{~A}) \rightarrow 420(\mathrm{~B})$ \\
\hline $\mathrm{K} 1 \mathrm{~K} 2-($ AluI $) 2$ & $\begin{array}{l}330+130(\mathrm{~B}) \rightarrow 320+130(\mathrm{D}), \\
460(\mathrm{~A}), 325+130(\mathrm{C})\end{array}$ \\
\hline $\mathrm{K} 1 \mathrm{~K} 2-($ Hinfl $) 1$ & $230+255(\mathrm{~B}) \rightarrow 485(\mathrm{~A})$ \\
\hline $\mathrm{K} 1 \mathrm{~K} 2-($ Hinfl $) 2$ & $380(\mathrm{~A}) \rightarrow 370(\mathrm{~B})$ \\
\hline $\mathrm{K} 1 \mathrm{~K} 2-($ Hinfl $) 3$ & $340(\mathrm{C}) \rightarrow 450(\mathrm{~A}), 360(\mathrm{~B})$ \\
\hline $\mathrm{K} 1 \mathrm{~K} 2-($ Hinfl $) 4$ & $190(\mathrm{~B}) \rightarrow 200(\mathrm{~A})$ \\
\hline $\mathrm{K} 1 \mathrm{~K} 2-(\operatorname{Taq} \mathrm{I}) 1$ & $700(\mathrm{~A}) \rightarrow 670(\mathrm{~B})$ \\
\hline $\mathrm{K} 1 \mathrm{~K} 2-(\operatorname{Taq} \mathrm{I}) 2$ & $650(\mathrm{C}) \rightarrow 700(\mathrm{~A}), 655(\mathrm{~B}), 600(\mathrm{D})$ \\
\hline $\mathrm{K} 1 \mathrm{~K} 2-(\operatorname{Taq} \mathrm{I}) 3$ & $340(\mathrm{~A}) \rightarrow 335(\mathrm{~B}), 270(\mathrm{C})$ \\
\hline $\mathrm{K} 1 \mathrm{~K} 2-(\operatorname{Taq} \mathrm{I}) 4$ & $265(\mathrm{~A}) \rightarrow 250(\mathrm{D}), 260(\mathrm{~B}), 255(\mathrm{C})$ \\
\hline
\end{tabular}

${ }^{\mathrm{z}} \mathrm{A}, \mathrm{B}, \mathrm{C}, \mathrm{D}, \mathrm{E}, \mathrm{F}, \mathrm{G}$, and $\mathrm{H}$ represent different polymorphisms in each polymorphic site.

and K1K2-Hinfl and K1K2-TaqI showed four polymorphic sites (Table 3 ). Of the 23 polymorphic sites, 13 showed three or more patterns and the most polymorphic were $\mathrm{HK}-(A l u \mathrm{I}) 2$ and $\mathrm{HK}-($ Hinfl $) 1$ with 8 and 6 different patterns, respectively. The rest of the polymorphic sites presented only two different restriction patterns (Table 3 ).

The restriction patterns obtained in the combination $\mathrm{K} 1 \mathrm{~K} 2-$ Hinf in 11 interspecific hybrids are shown in Fig. 1. The first interspecific hybrid shows a different restriction pattern from the rest with the presence of a fragment of $\approx 485 \mathrm{bp}$, which is substituted by two fragments of 230 and $255 \mathrm{bp}$ in the rest of the samples, incited by a point mutation (Table 3). Also, most of the interspecific hybrids show a fragment of $\approx 340 \mathrm{bp}$, except the fourth and the fifth ones, which both show a fragment of $450 \mathrm{bp}$ and the seventh one, which shows a $360 \mathrm{bp}$ fragment. This kind of polymorphism was probably due to indel mutations.

Chloroplast DNA haplotypes. The combination of all the mutations resulted in 33 cpDNA haplotypes (described in Table 4 ), which represent the 84 accessions studied as are shown in Table 1. Fourteen from the total cpDNA haplotypes were shared by two or more accessions, and 19 were unique haplotypes to their respective accessions. Twenty-seven haplotypes were specific to only one Prunus species, while the remaining six (H1, H3, H4, H9, H18, and H20) were shared by two different

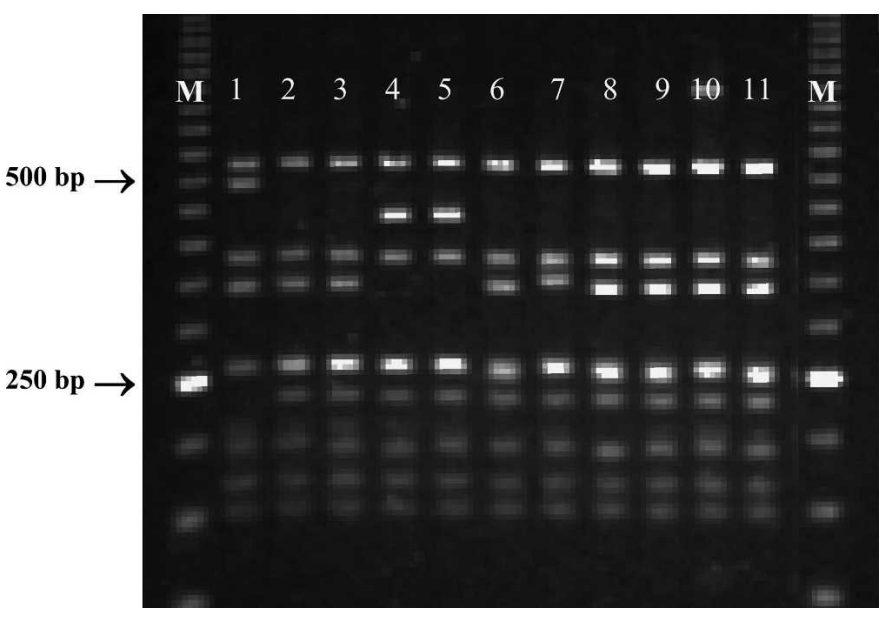

Fig. 1. Restriction patterns obtained on agarose gel with the primer pairrestriction enzyme combination $\mathrm{K} 1 \mathrm{~K} 2-\operatorname{Hinf} \mathrm{I}$ in 11 interspecific hybrids of Prunus; $\mathrm{M}=$ molecular size marker (50-bp ladder; Amersham, Piscataway, NJ). The first interspecific hybrid shows a different restriction pattern with the presence of a fragment of $\approx 485 \mathrm{bp}$, which is substituted by two fragments of 230 and $255 \mathrm{bp}$ in the remaining samples, incited by a point mutation. Most of the interspecific hybrids show a fragment of $\approx 340 \mathrm{bp}$ except the fourth and the fifth ones, which both show a fragment of $450 \mathrm{bp}$, and the seventh one, which shows a 360-bp fragment. This kind of polymorphism was probably due to indel mutations.

species (Table 1). On the other hand, 15 from the 24 species analyzed (directly or through their interspecific hybrids) presented only one haplotype, while eight species $[P$. americana Marshall, P. besseyi L.H. Bailey, P. dasycarpa Ehrh., P. kansuensis Rehd., P. incana (Pall.) Batsch, P. prostrata Labill., $P$. pumila L., and $P$. spinosa] presented two different haplotypes. Prunus cerasifera Ehrh. is the unique species of this study and showed eight different haplotypes (Table 1).

Haplotype $\mathrm{H} 1$ was the most abundant and was shared by 14 accessions (16.1\%): 13 were interspecific hybrids with $P$. pumila or $P$. besseyi as female parents, and the fourteenth one was a parent representing the $P$. besseyi species (Table 1). Other hybrid accessions with $P$. besseyi as the female parent shared the haplotype $\mathrm{H} 20$ that had an interspecific hybrid with $P$. incana as its maternal progenitor (Table 1).

Haplotype $\mathrm{H} 2$ was only shared by seven accessions: six were interspecific hybrids showing $P$. salicina as the female parent, and the last one represented the species $P$. salicina itself. Similar results were found for haplotype $\mathrm{H} 7$, which was detected in only two interspecific hybrids with $P$. brigantiaca Vill. as female parent and the species $P$. brigantiaca itself. Moreover, haplotype H6 was detected in two interspecific hybrids with $P$. kansuensis as the female parent and the species $P$. kansuensis itself, but this species was also represented by another haplotype, H17 (Table $1)$. The $P$. americana accession and two interspecific hybrids with $P$. americana as female parent shared the same haplotype H14. This species also showed haplotype H8 (Table 1).

All interspecific hybrid accessions with $P$. dasycarpa as female parent shared the same haplotype H3 with some hybrid accessions presenting $P$. cerasifera as the female parent. The $P$. dasycarpa species also showed the haplotype H31. Moreover, $P$. cerasifera presented six other haplotypes (H5, H10, H15, H16, H19, and H23) and shared the haplotype H18 with $P$. prostrata (Table 1). Haplotype $\mathrm{H} 4$ was common to all the accessions presenting $P$. fruticosa or $P$. cerasus as the female 


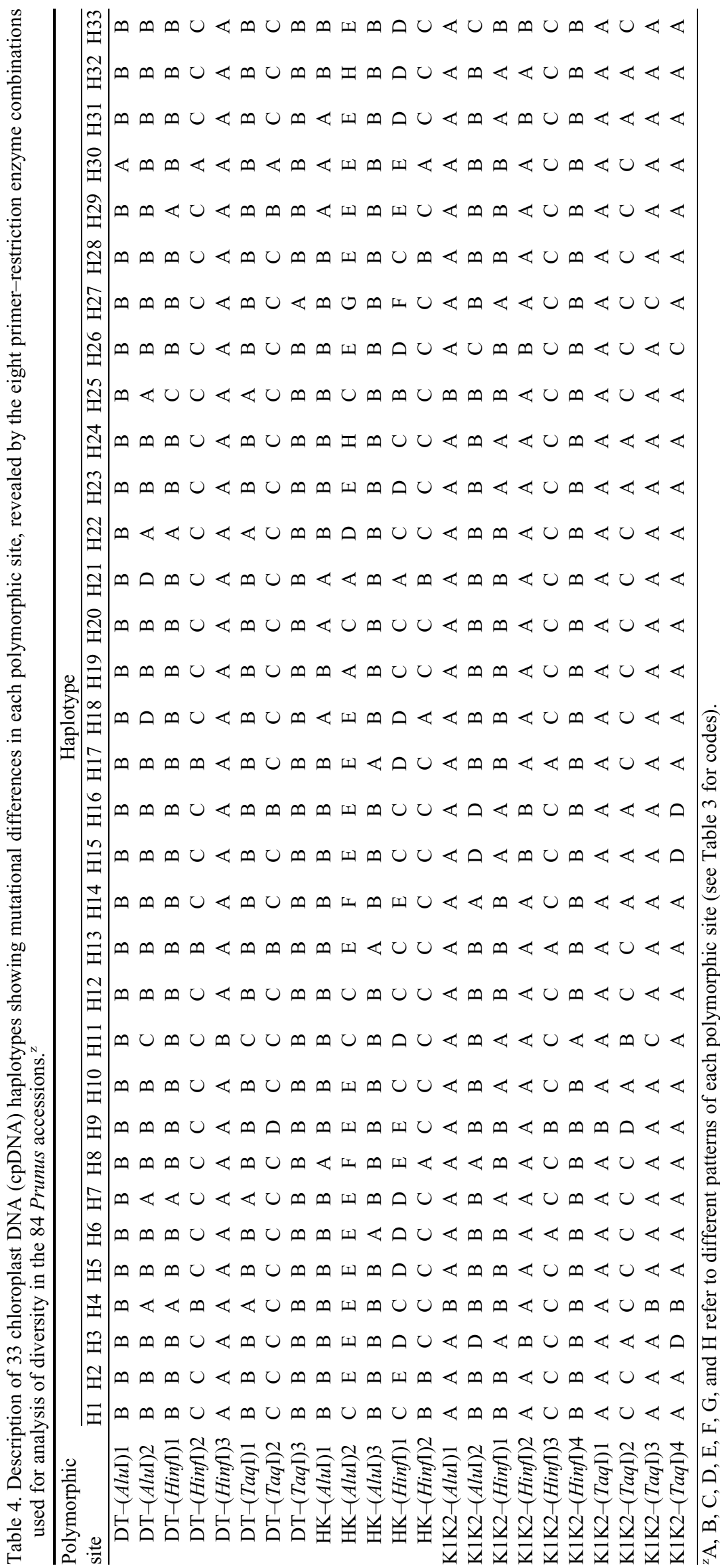


parent and to the species $P$. fruticosa itself. The $P$. tenella Batsch accession and two interspecific hybrids with $P$. tenella or $P$. nana (L.) Stokes as female parent were included under the same haplotype, H9.

\section{Chloroplast DNA diversity and genetic relationships in Prunus}

UPGMA ANALYSIS. The genetic diversity among the different cpDNA haplotypes was shown in the UPGMA cluster analysis. At a similarity coefficient value of 0.82 , the phenogram showed two main groups, and three haplotypes (H9, H11, and H27) appeared out of the groups (Fig. 2).

The first main group comprised haplotypes of Prunus species belonging to the subgenera Prunophora, Amygdalus, and Cerasus section Microcerasus Webb. The second main group was clustered to the first one at a similarity value of 0.77 and was composed of four haplotypes, three representing species of the subgenus Cerasus section Eucerasus Koehne (H4), section Mahaleb Focke (H22), and section Pseudocerasus Koehne (H25), and the fourth haplotype (H7) was present in $P$. brigantiaca species, member of subgenus Prunophora section Armeniaca (Mill.) Koch. Two of the three haplotypes clustered out of the groups were represented by $P$. spinosa species (H27 and H11), while the third one (H9) represented the cpDNA type of $P$. tenella and $P$. nana species.

In the first main group, three subgroups were observed (Fig. 2). A close relationship was detected among haplotypes included in the first subgroup, representing species of the subgenus Prunophora sections Armeniaca, Euprunus Koehne,

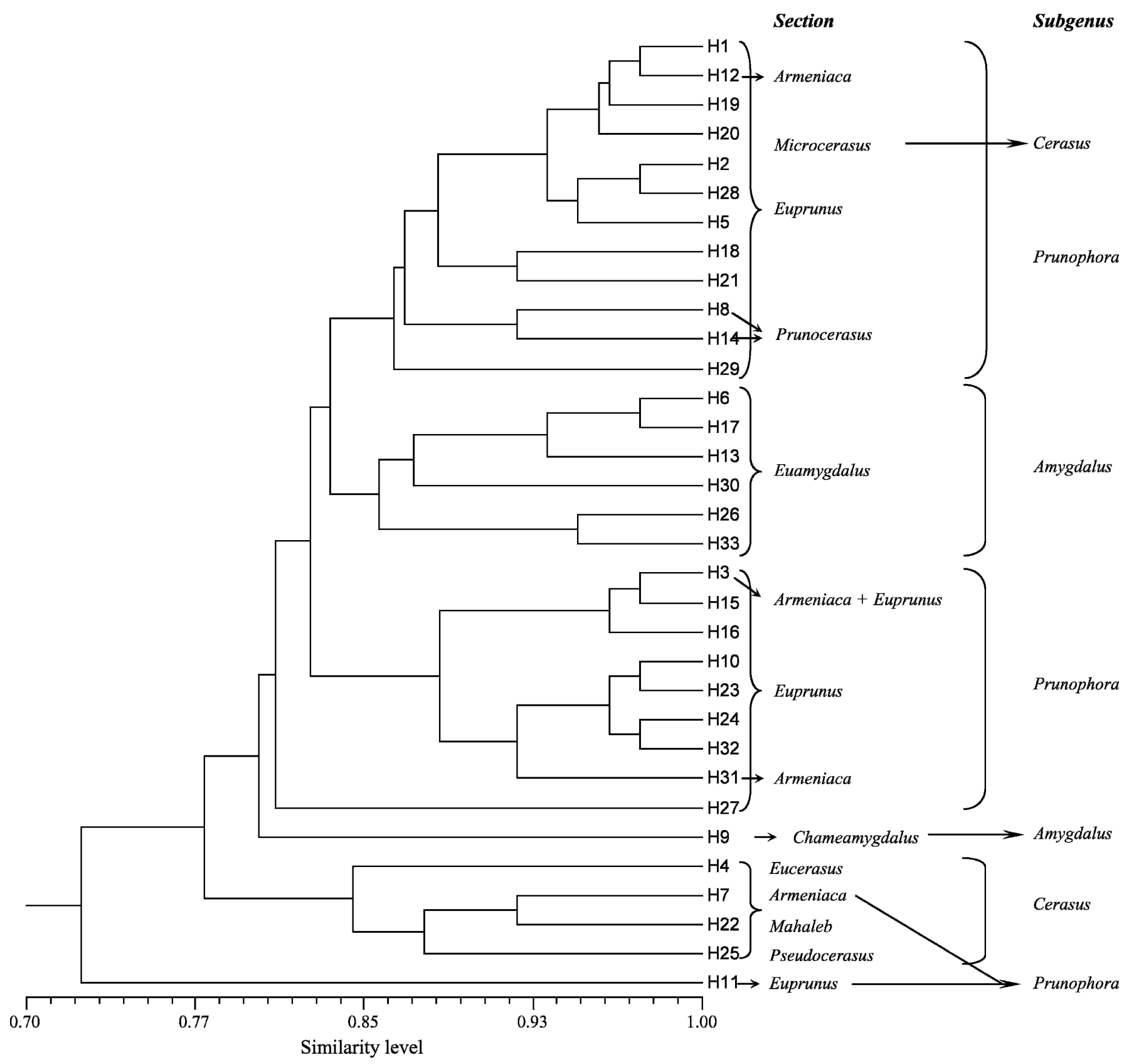

Fig. 2. Dendrogram of 33 Prunus chloroplast DNA (cpDNA) haplotypes (H1-H33, described in Table 4) generated by UPGMA clustering analysis using the simple matching coefficient. Classification into subgenera and sections is according to Rehder (1940). 
and Prunocerasus Koehne, and species of the subgenus Cerasus section Microcerasus (H1, H20, H21, H28, and H29; see Table 1). Haplotypes representing species of the subgenus Amygdalus section Euamygdalus Spach. were clustered together under the second subgroup (Fig. 2). The third subgroup, clustered at a similarity value of 0.82 from the previous two, contained haplotypes, which mostly represented species of subgenus Prunophora section Euprunus. Haplotypes representing species of section Armeniaca (H3 and H31) were also included in this subgroup.

Minimum-Length SPANNing TREe

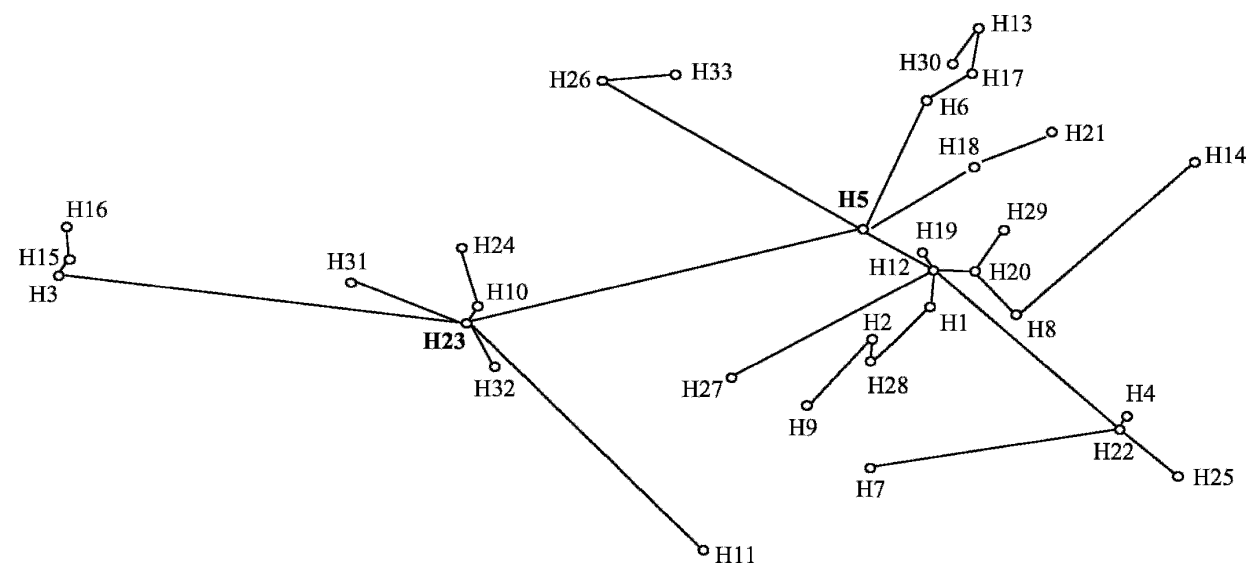

Fig. 3. Minimum-length spanning tree of 33 chloroplast DNA (cpDNA) haplotypes (H1 to H33, described in Table 4) from the 84 Prunus accessions studied.

ANALYSIS. The relationships among the 33 cpDNA haplotypes were also presented in a minimumlength spanning tree (MST) shown in Fig. 3. The MST offers a good representation of linked haplotype groups based on the mutational differences among them. Two main clusters of haplotypes could be observed with $\mathrm{H} 23$ and $\mathrm{H} 5$ as the internal nodes. The cluster linked to H23 contained haplotypes representing species of subgenus Prunophora section Euprunus and section Armeniaca for P. dasycarpa (H3, H31) species. The cluster linked to H5 was composed of haplotypes representing species of subgenera Amygdalus, Cerasus, and Prunophora.

\section{Discussion}

Chloroplast DNA haplotypes: inheritance AND RELATIONSHIPS. Assessment of cpDNA variation in 84 accessions representing 62 interspecific hybrids and 22 species of Prunus revealed 33 different cpDNA haplotypes. Twentyseven from the total haplotypes observed were specific to a unique species (Table 1), which is very interesting for further molecular characterization of Prunus species. Considering that chloroplast DNA is maternally inherited in most angiosperms, assessment of cpDNA variation in Prunus accessions provides good information on their maternal relationships.

Haplotype $\mathrm{H} 2$ was shared by the species $P$. salicina and six complex interspecific hybrids of Prunus which have $P$. salicina as maternal progenitor (Table 1). The same case was observed for some interspecific hybrids represented by other six haplotypes (H1, H4, H6, H7, H9, and H14), which had the same haplotypes as their maternal progenitors (Table 1). These results seem to agree with the maternal inheritance of cpDNA in Prunus and, in some cases, permit identity confirmation of the maternal progenitor. However, some interspecific hybrids showed different haplotypes from the expected maternal ones. This fact was observed when the Prunus species analyzed directly or through their interspecific hybrids presented two or more different haplotypes, as $P$. americana, $P$. besseyi, P. dasycarpa, $P$. kansuensis, $P$. incana, $P$. prostrata, $P$. pumila, $P$. spinosa, and $P$. cerasifera. This last species is the only one in this study that showed eight different haplotypes, probably due to the large number of $P$. cerasifera accessions (16) analyzed (directly or through their interspecific hybrids) (Table 1). The mentioned discordances could be due to the existence of

intraspecific cpDNA variation, which has been previously reported in Prunus species such $P$. spinosa and $P$. avium (Mohanty et al., 2000, 2001, 2002). Therefore, to confirm the maternal origin of interspecific hybrids based on their cpDNA haplotypes, it will be necessary to analyze more accessions of these Prunus species to determine better their intraspecific cpDNA diversity.

Several pairs of Prunus species shared the same cpDNA haplotypes ( $\mathrm{H} 1, \mathrm{H} 3, \mathrm{H} 4, \mathrm{H} 9, \mathrm{H} 18$, and H20; Table 1). The predominant haplotype $\mathrm{H} 1$ was presented in the $P$. besseyi species accession and in 13 hybrids of Prunus having $P$. pumila or $P$. besseyi as female parent (Table 1). If it is assumed that cpDNA is maternally inherited in Prunus, then the obtained result provides information about the existence of a maternal relationship between $P$. pumila and $P$. besseyi. In fact, these two species are members of the same subgenus Cerasus section Microcerasus as defined by Rehder (1940). Close relationships between $P$. pumila and $P$. besseyi were also reported by Mowrey and Werner (1990) using isozyme markers.

On the other hand, nine interspecific hybrid accessions presented in common the haplotype H3; these hybrids have $P$. cerasifera or $P$. dasycarpa as female parents. This result would indicate a close maternal relationship between these two species. Prunus dasycarpa is, indeed, a naturally occurring hybrid of $P$. cerasifera and $P$. armeniaca (Mehlenbacher et al., 1990). Moreover, these authors reported that $P$. cerasifera $\times P$. armeniaca produces hybrids resembling the natural interspecific hybrid species $P$. dasycarpa when plum is used as the female parent. According to these findings and the results obtained in this study, we can suggest that $P$. cerasifera is the maternal progenitor of $P$. dasycarpa.

Haplotype $\mathrm{H} 4$ was shared by six accessions: five are hybrids which have $P$. fruticosa or $P$. cerasus as female parents, and one represents the $P$. fruticosa itself. The grouping of these six accessions under the same haplotype $\mathrm{H} 4$ could be due to the existence of close maternal relationships between $P$. fruticosa and $P$. cerasus. In fact, these two species belong to the same subgenus Cerasus section Eucerasus. In addition, sour cherry ( $P$. cerasus) is considered to be the product of interspecific hybridization of sweet cherry $(P$. avium $)$ and ground cherry (P. fruticosa) (Olden and Nybom, 1968). According to Mehlenbacher et al. (1990), crossings between ground cherry and diploid or tetraploid sweet cherry selections gave progenies 
resembling sour cherry. Moreover, the study of Panda et al. (2003) confirms that $P$. avium is not the maternal parent of $P$. cerasus. Our findings are in agreement with these reports, suggesting that $P$. fruticosa may be the female parent of P. cerasus.

Haplotype H9 was shared by three accessions: two are hybrids having $P$. tenella or $P$. nana as female parent, and one represents the species $P$. tenella (Table 1). According to Rehder (1940), P. tenella and $P$. nana are synonyms, which can explain their cpDNA pattern similarity.

Chloroplast DNA diversity and Genetic Relationships IN Prunus. The cpDNA data obtained from the PCR-RFLP analysis of the 84 Prunus accessions were used to produce a dendrogram by the UPGMA cluster method and a MST. Comparison of the cpDNA phenogram with the taxonomic classification of Prunus based on morphological data (Rehder, 1940) shows similarities but also several apparent discrepancies.

In the UPGMA phenogram (Fig. 2), the first main group contained all haplotypes representing species of subgenus Cerasus section Microcerasus, which clustered with haplotypes representing species of subgenera Prunophora and Amygdalus. The remaining haplotypes representing species of subgenus Cerasus, sections Eucerasus (H4), Mahaleb (H22) and Pseudocerasus (H25), were clustered in the second main group. Therefore, the traditional classification of Microcerasus included within subgenus Cerasus (Rehder, 1940) was not supported by this UPGMA analysis. Moreover, Bortiri et al. (2001) and Lee and Wen (2001) also noted that section Microcerasus does not show any relationship to the rest of the subgenus Cerasus. Likewise, Katayama and Uematsu (2005) found that Amygdalus, Prunophora, and Cerasus section Microcerasus formed a major group in the UPGMA tree performed using RFLP data from chloroplast DNA. A similar relation among haplotypes representing Microcerasus species and haplotypes representing the rest of species of the subgenus Cerasus was also shown in the MST analysis (Fig. 3).

The first main group in the UPGMA phenogram was composed of three subgroups (Fig. 2). The first subgroup included species of section Microcerasus and species of subgenus Prunophora. This subgroup showed the close relatedness of $P$. pumila and $P$. besseyi $(\mathrm{H} 1, \mathrm{H} 20$, and $\mathrm{H} 28)$ to plums $[P$. salicina and $P$. cerasifera $(\mathrm{H} 2, \mathrm{H} 5$, and $\mathrm{H} 19)]$, and to apricot $[P$. armeniaca (H12)]. Ramming and Cociu (1990) reported that the cherry species $P$. bessey $i$ and $P$. pumila appeared more closely related to plums than to cherries. Bortiri et al. (2001) mentioned that section Microcerasus was not monophyletic and that most of its species might be more closely related to the subgenus Prunophora than to subgenus Cerasus. More research is required to find the closest relatives belonging to Microcerasus species.

Haplotypes representing species of the subgenus Amygdalus section Euamygdalus were grouped together in the second subgroup (Fig. 2). The P. kansuensis, $P$. persica, and $P$. davidiana (Carriere) Franch. peach species examined in this study, and represented by haplotypes H6, H17, H13, and H30 (Table 1), clustered together at a high similarity value (0.86). The P. bucharica (Korsch.) Hand-Mazz. (H26) and P. dulcis (H33) almond species were closely related at a similarity value of 0.94 , and they were also joined to peach species. In fact, members of section Euamygdalus were often classified as either almond-like or peach-like plants (Scorza and Okie, 1990), which is well supported by our findings. The MST (Fig. 3) also showed close relationships among Amygdalus haplotypes (section Euamygdalus) and was in agreement with the UPGMA clustering. Nevertheless, the $P$. tenella member of subgenus Amygdalus section Chameamygdalus Spach., represented by haplotype H9, appears out of the Amygdalus group (Figs. 2 and 3 ). This result indicates that the cpDNA of $P$. tenella can be highly divergent from the other Amygdalus species. Kester et al. (1990) reported that the section Chameamygdalus includes species genetically divergent from species of other almond sections and that it rarely crosses with cultivated almond.

The close relationships showed between H24 ( $P$. insititia) and H32 (P. domestica) (Fig. 2) was also reported by Bouhadida et al. (2005) and Casas et al. (1999) analyzing the genetic diversity of Prunus rootstocks with SSR and RAPD markers, respectively. These two species were closely related to $P$. cerasifera, represented by $\mathrm{H} 10$ and H23 (Figs. 2 and 3). Prunus domestica was thought to be derived from a crossing between a diploid $(2 n=2 x=16)$ cherry plum or myrobalan, $P$. cerasifera, and a tetraploid $(2 n=4 x=32)$ sloe or blackthorn, $P$. spinosa (Crane and Lawrence, 1952). This hypothesis has been widely accepted by horticulturists and crop plant evolutionists. However, Zohary (1992) reported that $P$. domestica probably evolved directly from the variable $P$. cerasifera, because of the close morphological similarities between them, and the wide morphological divergence between these two plums and $P$. spinosa. According to the UPGMA dendrogram and the MST analyses (Figs. 2 and 3), haplotypes H11 and H27 representing the $P$. spinosa plum species appear separated from haplotypes representing other plum species.

Prunus fruticosa and $P$. cerasus species (haplotype H4) were grouped together with $P$. brigantiaca $(\mathrm{H} 7), P$. mahaleb (Dougl.) L. (H22), and P. lannesiana (Carr.) E.H. Wilson (H25) (Figs. 2 and 3). An apparent common maternal inheritance may exist among these species. This result can be explained by the fact that all species of this group belong to the subgenus Cerasus with the exception of $P$. brigantiaca, which belong to subgenus Prunophora section Armeniaca. According to the phylogenetic studies of Mowrey and Werner (1990) based on isozymes, $P$. mahaleb a member of the section Mahaleb was clustered together with $P$. cerasus and $P$. fruticosa, which were classified within section Eucerasus. Bortiri et al. (2001) reported in their nuclear ITS and chloroplast DNA study that $P$. lannesiana was a synonym of $P$. serrulata Lindl. and that these species was closely related to $P$. fruticosa and $P$. cerasus. Our results are in agreement with these findings. The placement of European native apricot species $P$. brigantiaca $(\mathrm{H} 7)$ within this group obscured its relationship with respect to other apricots. Bortiri et al. (2001) also reported that P. brigantiaca was not included in the Armeniaca clade, and that the ITS sequence of this species had many ambiguities, some of them in regions of high variability. Additional studies including more accessions of Prunus species and using more molecular data (e.g., more combinations of primers and restriction enzymes) would be required to understand the true position of $P$. brigantiaca within the genus Prunus and to draw a more accurate phylogeny of Prunus.

In summary, the results of this work demonstrate the usefulness of cpDNA diversity detected using PCR-RFLP methods to analyze the genetic relationships among species and cultivars of Prunus. This study contributes to the understanding of the maternal inheritance of cpDNA in Prunus, and permits confirmation of the maternal progenitors of different hybrids of this genus. The maternal inheritance of cpDNA and 
the genetic relationships revealed by the cpDNA variation will be a useful tool in designing crosses for future breeding programs. The cpDNA analysis will also provide a starting point for a more detailed phylogenetic analysis of the genus Prunus.

\section{Literature Cited}

Arulsekar, S., D.E. Parfitt, and D.E. Kester. 1986. Comparison of isozyme variability in peach and almonds cultivars. J. Hered. 77:272-274.

Badenes, M.L. and D.E. Parfitt. 1995. Phylogenetic relationships of cultivated Prunus species from an analysis of chloroplast DNA variation. Theor. Appl. Genet. 90:1035-1041.

Bentham, G. and J.D. Hooker. 1865. Genera plantarum, Vol. 1. Reeve \& Co., London.

Bortiri, E., S.H. Oh, J. Jiang, S. Baggett, A. Granger, C. Weeks, M. Buckingham, D. Potter, and D.E. Parfitt. 2001. Phylogeny and systematics of Prunus (Rosaceae) as determined by sequence analysis of ITS and the chloroplast $\operatorname{trnL}-\operatorname{trnF}$ spacer DNA. Syst. Bot. 26:797-807.

Bouhadida, M., A.M. Casas, M.A. Moreno, and Y. Gogorcena. 2005. Genetic diversity of Prunus rootstocks using microsatellite markers. Acta Hort. 663:625-627.

Casas, A.M., E. Igartua, G. Balaguer, and M.A. Moreno. 1999. Genetic diversity of Prunus rootstocks analysed by RAPD markers. Euphytica 110:139-149.

Cheng, F.S., S.K. Brown, and N.F. Weeden. 1997. A DNA extraction protocol from various tissues in woody species. HortScience 32: 921-922.

Crane, M.B. and W.J.C. Lawrence. 1952. The genetics of garden plants. 4th ed. Macmillan, London.

Demesure, B., N. Sodzi, and R.J. Petit. 1995. A set of universal primers for amplification of polymorphic non-coding regions of mitochondrial and chloroplast DNA in plants. Mol. Ecol. 4:129-131.

Demesure, B., B. Comps, and R.J. Petit. 1996. Chloroplast DNA phylogeography of the common beech (Fagus sylvatica L.) in Europe. Evolution Int. J. Org. Evolution 50:2515-2520.

De Tournefort, J.P. 1700. Institutiones Rei Herbariae. Editio Altera, Gallica Longe Auctior, Quingentis Circiter Tabulis Aeneis Adornata, Typographia Regia, Paris.

Dosba, F., R. Bernhard, and A. Zanetto. 1994. Importance des ressources génétiques des Prunus. C.R. Acad. Agr. France 80:45-57. Dumolin-Lapegue, S., M.H. Pemonge, and R.J. Petit. 1997. An enlarged set of consensus primers for the study of organelle DNA in plants. Mol. Ecol. 6:393-397.

El Mousadek, A. and R.J. Petit. 1996. Chloroplast DNA phylogeography of the argan tree of Morocco. Mol. Ecol. 5:547-555.

Gielly, L. and P. Taberlet. 1994. The use of chloroplast DNA to resolve plant phylogenies: non-coding vs RbcL sequences. Mol. Biol. Evol. 11:769-777.

Ingram, C. 1948. Ornamental cherries. Country Life, London.

Kaneko, T., T. Terachi, and K. Tsunewaki. 1986. Studies on the origin of crop species by restriction endonuclease analysis of organellar DNA. II. Restriction analysis of ctDNA of 11 Prunus species. Jpn. J. Genet. 61:157-168.

Katayama, H. and C. Uematsu. 2005. Structural analysis of chloroplast DNA in Prunus (Rosaceae): evolution, genetic diversity and unequal mutations. Theor. Appl. Genet. 111:1430-1439.

Kester, D.E., T.M. Gradziel, and C. Grasselly. 1990. Almonds (Prunus), p. 699-758. In: J.N. Moore and J.R. Ballington (eds.). Genetic resources of temperate fruit and nut crops. International Society for Horticultural Science, Wageningen, The Netherlands.

King, R.A. and C. Ferris. 1998. Chloroplast DNA phylogeography of Alnus glutinosa (L.). Gaertn. Mol. Ecol. 7:1151-1161.

Koehne, E. 1893. Deutsche Dendrologie. Verlag von Ferdinand Enke, Stuttgart, Germany.
Koehne, E. 1911. Die Gliederung von Prunus subgen. Padus., Vol. 52, pp. 101-108. Verhandlungen des Botanischen Vereins der Provinz Brandenburg. Der Botanische Verein der Provinz Brandenburg, Berlin.

Konieczny, A. and F.M. Ausubel. 1993. A procedure for mapping Arabidopsis mutations using codominant ecotype-specific PCRbased markers. Plant J. 4:403-410.

Layne, R.E.C. 1987. Peach rootstocks, p. 185-216. In: R.C. Rom and R.F. Carlson (eds.). Rootstocks for fruit crops. Wiley, NewYork.

Lee, S. and J. Wen. 2001. A phylogenetic analysis of Prunus and the Amygdaloideae (Rosaceae) using ITS sequences of nuclear ribosomal DNA. Amer. J. Bot. 88:150-160.

Mehlenbacher, S.A., V. Cociu, and L.F. Hough. 1990. Apricots (Prunus), p. 63-173. In: J.N. Moore and J.R. Ballington (eds.). Genetic resources of temperate fruit and nut crops. International Society for Horticultural Science, Wageningen, The Netherlands.

Mohanty, A., J.P. Martín, and I. Aguinagalde. 2000. Chloroplast DNA diversity within and among populations of the allotetraploid Prunus spinosa L. Theor. Appl. Genet. 100:1304-1310.

Mohanty, A., J.P. Martín, and I. Aguinagalde. 2001. A population genetic analysis of chloroplast DNA in wild populations of Prunus avium L. in Europe. Heredity 87:421-427.

Mohanty, A., J.P. Martín and I. Aguinagalde. 2002. Population genetic analysis of European Prunus spinosa (Rosaceae) using chloroplast DNA markers. Amer. J. Bot. 89:1223-1228.

Moreno, M.A. 2004. Breeding and selection of Prunus rootstocks at the Aula Dei Experimental Station, Zaragoza, Spain. Acta Hort. 658:519-528.

Mowrey, B.D. and D.J. Werner. 1990. Phylogenetic relationships among species of Prunus as inferred by isozyme markers. Theor. Appl. Genet. 80:129-133.

Olden, E.J. and N. Nybom. 1968. On the origin of Prunus cerasus L. Hereditas 59:327-345.

Panda, S., J.P. Martín, and I. Aguinagalde. 2003. Chloroplast DNA study in sweet cherry cultivars (Prunus avium L.) using PCR-RFLP method. Genet. Resour. Crop Evol. 50:489-495.

Prim, R.C. 1957. Shortest connection networks and some generalizations. Bell Laboratories Tech. J. 36:1389-1401.

Ramming, D.W. and V. Cociu. 1990. Plums (Prunus), p. 233-287. In: J.N. Moore and J.R. Ballington (eds.). Genetic resources of temperate fruit and nut crops. International Society for Horticultural Science, Wageningen, The Netherlands.

Rehder, A. 1940. A manual of cultivated trees and shrubs hardy in North American exclusive of the subtropical and warmer temperate regions. 2nd ed. Macmillan, New York.

Rohlf, F.J. 2000. NTSYS-pc numerical taxonomy and multivariate analysis system. Version 2.1. Exeter Software, Setauket, NY.

Rohrer, J.R., R. Ahmad, S.M. Southwick, and D. Potter. 2004. Microsatellite analysis of relationships among North American plums (Prunus sect. Prunocerasus, Rosaceae). Plant Syst. Evol. 244:69-75. Scorza, R. and W.R. Okie. 1990. Peaches (Prunus), p. 175-231. In: J.N. Moore and J.R. Ballington (eds.). Genetic resources of temperate fruit and nut crops. International Society for Horticultural Science, Wageningen, The Netherlands.

Taberlet, P., L. Gielly, G. Pautou, and J. Bouvet. 1991. Universal primers for amplification of three non-coding regions of chloroplast DNA. Plant Mol. Biol. 17:1105-1109.

Uematsu, C., T. Sasakuma, and Y. Ogihara. 1991. Phylogenetic relationships in the stone fruit group of Prunus as revealed by restriction fragment analysis of chloroplast DNA. Jpn. J. Genet. 66:59-69.

Webster, A.D. 2005. The origin, distribution and genetic diversity of temperate tree fruits, p. 1-11. In: J. Tromp, A.D. Webster, and S.J. Wertheim (eds.). Fundamentals of temperate zone tree fruit production. Backhuys Publishers, Leiden, The Netherlands.

Zohary, D. 1992. Is the European plum, Prunus domestica L., a $P$. cerasifera Ehrh. $\times P$. spinosa L. allo-polyploid? Euphytica 60 : $75-77$. 